\title{
Knowledge Attitudes and Behavior of Parents towards Human Papillomavirus (HPV) Vaccine in Prevention of Cervical Cancer in Adolescent Girls in Botswana
}

\author{
Senatla KT* and Dolamo BL \\ University of South Africa, Department of Health Studies, South Africa \\ *Corresponding author: Kgola T Senatla, University of South Africa, Department of \\ Health Studies, South Africa, Email: kgolis71@gmail.com
}

\section{Research Article \\ Volume 2 Issue 10}

Received Date: September 20, 2018

Published Date: September 29, 2018

\section{Abstract}

The purpose of the study was to determine the knowledge, attitude and behaviour of parents towards HPV and HPV vaccine in preventing cervical cancer in girls aged 9 to 13 years and HPV vaccine uptake in Sefhare, Botswana. A descriptive cross-sectional study was conducted in 2017 among 100 parents of adolescent girls of ages 9-13 years living in four wards of Sefhare village. Data was collected using a questionnaire in face-to-face interviews. Data was analysed using SPSS version 19.The study found a high level (71.8\%) of knowledge about HPV infection, cervical cancer and HPV vaccine and a positive attitude (81.5\%) and behaviour (62\%) towards the HPV vaccine.

Keywords: Adolescent; Attitude; Behaviour; Cervical Cancer; Human Papilloma Virus (HPV); HPV Vaccine; Knowledge; Parents

\section{Introduction}

Human papillomavirus (HPV) is the most common cause of sexually-transmitted diseases (STDs) in men and women. It is also the established causative agent of cervical cancer, anal and penile cancers as well as genital warts in both men and women. Protection against strains of HPV that lead to cervical cancer can be achieved through immunisation with the HPV vaccine.

It is estimated that globally, there are 500,000 new cases of cervical cancer and 274,000 deaths occur annually $80 \%$ of the deaths occur in developing countries, where cervical cancer is the leading cause of cancer deaths among adult women $[1,2]$. Cervical cancer is a serious health problem that claims the lives of many women and remains a grave concern in Botswana and around the world [3]. It was estimated that 2,800 new cases of cervical cancer were diagnosed in France in 2011, leading to 1,000 deaths [4]. In a study in North India it was found that persistent HPV infection led to invasive cervical cancer in $80 \%$ of sexually active women [5].

HPV vaccine was first approved in the United States of America in 2006. By 2014 more than 57 countries had included the HPV vaccine in their national health programmes [6]. Cervarix (bivalent) and Gardasil (quadrivalent) vaccines are used for preventing HPV infections. Clinical trials indicated that three vaccine doses provide $90 \%$ to $100 \%$ protection against cervical infection and pre-cancer related to HPV 16 and 18 [6].

HPV, the vaccine is given to adolescent girls before sexual debutto maximise effectiveness. The HPV vaccine 


\section{Virology \& Immunology Journal}

is given in three doses. The first dose is given at first contact with the adolescent. The second dose is given two months after the first dose and the third dose is given four months following the second dose. The girl must receive all the three doses in order to be fully protected.

In Botswana it is estimated that 250 women are diagnosed with cervical cancer annually and 111 of them die [5]. Cervical HPV infection accounts for 53\% of women tested (95\% Confidence Interval [CI]) [7]. The HPV vaccine was licensed in Botswana in 2009, and is now given free to adolescent girls aged 9 to 13 years as a preventive strategy against HPV. The programme targets girls at primary school and out-of-school aged 9 to 13 years. The roll-out started in March 2013 and girls receive three doses of the vaccine knowledge, attitudes and behaviour towards HPV and HPV vaccine.

\section{Methodology}

A quantitative, descriptive and cross-sectional design was conducted. An anonymous questionnaire was used to collect data from the respondents. Data was collected at one point in time from each respondent. The study described how much the parents knew about HPV and the HPV vaccine as well as their attitudes and behaviour towards HPV and the vaccine. The study was approved by the Unisa Ethical Committee for Higher Degrees and Ministry of Health and Wellness, Botswana. The respondents signed the consent prior to the interview.

\section{Framework}

The study was guided by revised Health Belief Model as the theoretical framework for the study. Becker's Health Belief Model explains people's health-related behaviour, such as health care use and compliance with a medical regimen. The model states that health-seeking behaviour is influenced by a person's perception of a threat posed by the health problem, and the value associated with actions aimed at reducing the threat [8].

In this study the model explained parents' behaviour in relation to HPV vaccine uptake and their compliance as evidenced by completion of three doses.

Parents perceived cervical cancer as a serious disease, and knew that HPV causes cervical cancer, so they were willing to vaccinate their daughters. Some of girls were already vaccinated. Parents also believed that they were some health benefits of HPV vaccine therefore they allowed their daughters to be vaccinated. Accessing the
HPV vaccine was not a problem since the school nurse followed the girls to school for vaccination. Media radio/television reinforced the distributing the message of HPV vaccine. Nurses also gave a reminder before they go to school for subsequent vaccination of the girls.

\section{Study Population and Sampling}

The study population consisted of hundred (100) parents/guardians of adolescent girls aged 9-13 years in Sefhare. Both male and female parents were included in the study. Most of the adolescent at this age are attending primary schools doing standard $4-7$. The inclusion and exclusion criteria were based on the respondents' ability to participate in the study and design consideration. Consideration was also given to the subjects' ability to comprehend the study information and the legal competence to give informed consent. The study excluded parents below 18 years of age and those with mental impairment. Parents from four wards participated in the study; ward 1 . Ward 2, ward 3 and ward 4 respectively. Stratified systematic sampling was used in selection of wards. Simple random method of every fifth house was selected from each ward. And twenty-five households participated from each ward.

\section{Data Collection}

Data was collected by face-to-face interview, using structured questionnaire. The interviews were conducted at the respondent's homes. The researcher explained the purpose of the study and that participation was voluntary and they could withdraw anytime if they wish to do so. The respondents signed the consent form prior to the interview.

\section{Data Collection Instrument}

The researcher developed the questionnaire, and was approved by UNISA Ethical Committee for Higher degrees and Ministry of Health and Wellness (Botswana).

The instrument was tested and altered based on the feedback and limitations identified during the pilot study. The questionnaire comprised of the following sections:

- Section 1 - Demographic data: Respondents' age, gender, educational level and ward

- Section 2 - Knowledge of HPV: Respondents' knowledge of HPV infection, cervical cancer and the HPV vaccine

- Section 3 - Attitudes: Respondents' attitudes towards HPV vaccine

- Section 4 - Behaviour: Respondents' behaviour regarding HPV vaccination. 


\section{Virology \& Immunology Journal}

\section{Statistical Analysis}

Key variables were knowledge of HPV infection, cervical cancer and /HPV vaccine, attitudes towards HPV vaccine and behaviour towards HPV vaccine. Data was analysed using SPSS version 19.ANOVA was used to test relationship between the respondents' level of education and attitudes. It was also used to test relationship between respondents' level of education and behaviour. Percentages of parents' knowledge, attitudes and behaviour between wards was calculated and compared.

\section{Results}

\section{Demographic Characteristics}

The study comprised of hundred respondents, 78\% of whom were females while $22 \%$ were males. The age distribution ranged from 25 to 45 years and above. The results show that nearly half of the respondents $44 \%$ were of ages $40-44 y$ ears followed by $25 \%$ of $35-39$ years, $15 \%$ of $30-34,10 \%$ of $25-29$ and $6 \%$ above 45 respectively. Sixty-one (61 \%) of the respondents were single, $28 \%$ married, $5 \%$ divorced and $6 \%$ widowed. of the respondents, $40 \%(n=40)$ had attained secondary school. And 27\% had tertiary education. Almost half of the respondents (49\%) reported that they were unemployed, 43\% employed and $8 \%$ self-employed.

\section{Knowledge HPV Infection}

Most of respondents (76\%) had heard about HPV infection and $54 \%$ knew that it can be transmitted through sex, $27 \%$ were not sure.

A reasonable number, $68 \%$ knew that HPV infection can cause cervical cancer. Seventy-one (71\%) knew that knew that HPV infection can be prevented while $26 \%$ had no idea. Figure 2 depicts all the information about respondents' knowledge regarding HPV infection.

\begin{tabular}{|c|c|c|c|}
\hline & & Frequency & Percent (\%) \\
\hline \multirow{3}{*}{ Gender } & Male & 22 & 22 \\
\hline & Female & 78 & 78 \\
\hline & Total & 100 & 100 \\
\hline \multirow{6}{*}{ Age } & $25-29$ years & 10 & 10 \\
\hline & 30-34 years & 15 & 15 \\
\hline & 35-39 years & 25 & 25 \\
\hline & 40-44 years & 44 & 44 \\
\hline & 45 and above & 6 & 6 \\
\hline & Total & 100 & 100 \\
\hline \multirow{5}{*}{ Level of education } & Never been to school & 4 & 4 \\
\hline & Primary & 29 & 29 \\
\hline & Secondary & 40 & 40 \\
\hline & Tertiary & 27 & 27 \\
\hline & Total & 100 & 100 \\
\hline \multirow{4}{*}{ Occupation } & Unemployed & 49 & 49 \\
\hline & Self-employed & 8 & 8 \\
\hline & Employed & 43 & 43 \\
\hline & Total & 100 & 100 \\
\hline \multirow{5}{*}{ Marital status } & Single & 61 & 61 \\
\hline & Married & 28 & 28 \\
\hline & Divorced & 5 & 5 \\
\hline & Widowed & 6 & 6 \\
\hline & Total & 100 & 100 \\
\hline
\end{tabular}

Table 1: Respondents' demographic data. 


\section{Knowledge about Cervical Cancer}

Almost all (98\%) of respondents had heard about cervical cancer, $76 \%$ heard from health worker, $10 \%$ from friend and the other $10 \%$ heard from media. All who heard about cervical cancer believed that it is a serious disease and case death. Ninety-one (91\%) knew that cervical cancer is caused by HPV and $94 \%$ knew that it can be prevented. Ninety-three (93\%) knew that cervical cancer can be treated if diagnosed early. Slightly above half $(54 \%)$ of the respondents knew that Pap smear is

\section{Virology \& Immunology Journal}

done 2-3 years. Figure 3 shows respondents' knowledge about cervical cancer.

\section{Knowledge about HPV vaccine}

Most of the respondents (80\%) had heard about HPV vaccine. Fifty-nine (59\%) heard from the health worker while $16 \%$ heard from media and 12\% heard from a friend respectively. A large percentage $(80 \%)$ knew that $\mathrm{HPV}$ is used to prevent cervical cancer, and $30 \%$ knew the dosage intervals. Knowledge about HPV vaccine is shown in figure 4.

Have you heard about HPV infection?

Can HPV infection be transmitted through sex?

Does HPV infection cause cervical cancer?

Does using a condom partially protect one from contracting HPV infection?

Anyone who has had sex could contract HPV infection

Can HPV infection be prevented?

Do multiple sex partners increase the risk of acquiring HPV?

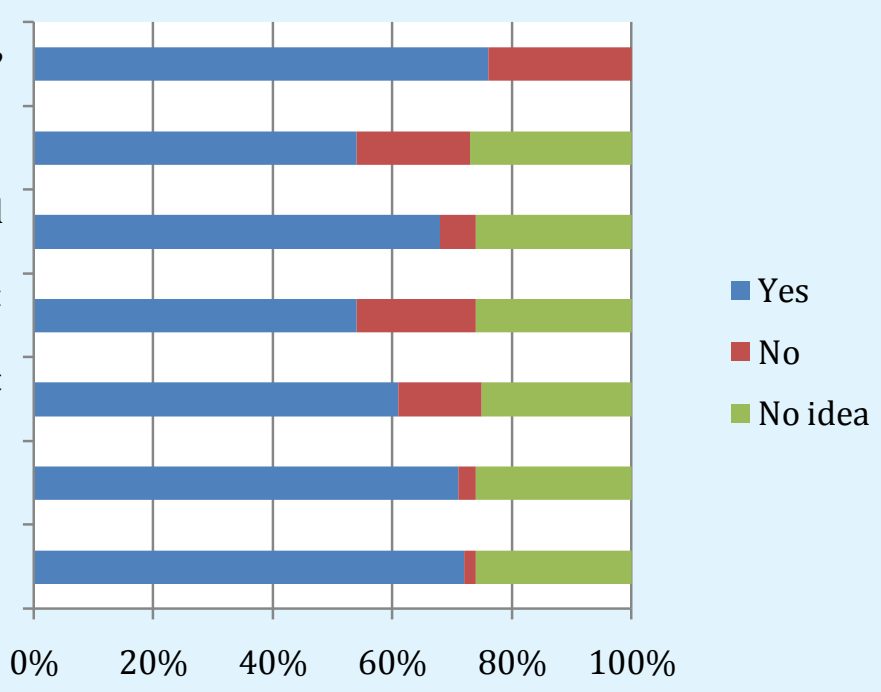

Figure 1: Respondests knowledge about HPV infection.

Have you heard about cervical cancer?

Is cervical cancer a serious disease?

Cervical cancer can cause death

Cervical cancer is caused by HPV

Cervical cancer can be prevented

Pap smear is used to detect cervical cancer

Pap smear is done every 2-3 years

Cervical cancer can be treated if..

Can cervical cancer threaten the..

Do you know anyone that has/had..

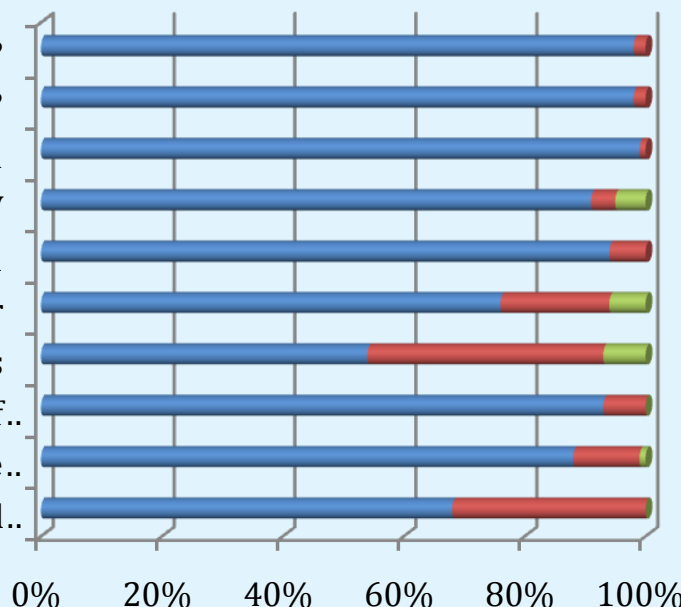

- Yes

no

No idea

Figure 2: Respondents' knowledge about cervical cancer. 


\section{Virology \& Immunology Journal}

Have you heard about HPV vaccine?

HPV vaccine is used to prevent cervical cancer

Do you know the dosage intervals of the the vaccine?

Have you been told about the benefits of a girl being vaccinated

Have the adverse effects been explained to you?

Do you know that the government ofers HPV vaccine for free?

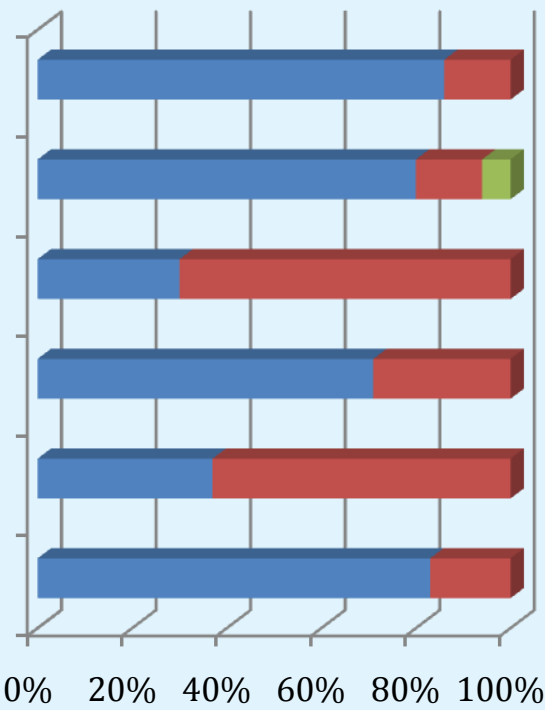

- Yes

No

No idea

Figure 3: Respondents' knowledge about the HPV vaccine.

\section{Attitudes towards HPV Vaccine}

All the respondents agreed that they felt confident in taking their daughters for vaccination; this was shown by $75 \%$ strongly agreeing and 25\% agreeing. Seventy-eight (78\%) also strongly agreed that there are health benefits for getting HPV vaccine whereas $22 \%$ agreed. Regarding whether HPV vaccine is best administered before sexual intercourse $55 \%$ strongly agreed while $32 \%$ agreed. As for the HPV vaccine information, 31\% agreed, $38 \%$ disagreed and $2 \%$ strongly disagreed that it was clearly explained. Figure 5 illustrate the respondents' attitudes towards HPV vaccine.

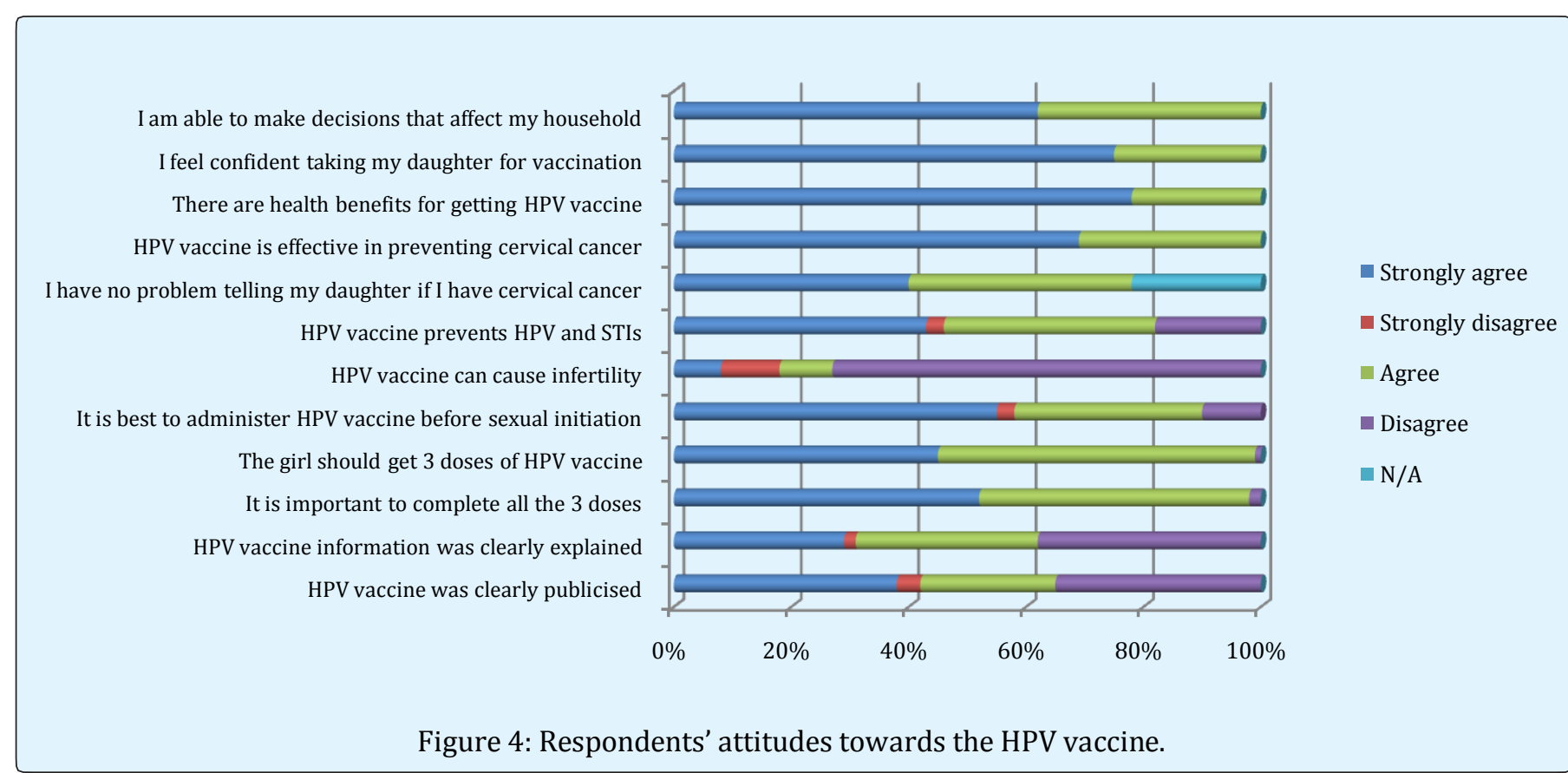

Senatla KT and Dolamo BL. Knowledge Attitudes and Behavior of Parents towards Human 
One-way ANOVA was used to test the relationship between the respondents' level of education and attitudes towards the HPV vaccine. $p$-value was calculated to evaluate the relationship. The question 'HPV is effective in preventing cervical cancer' obtained a significance of 0.074 . A significance of 0.036 was seen in the question 'the girl should get three doses of HPV vaccine'. For the question 'it is important to complete all 3 doses' a significance of 0.008 was obtained. A slight significance of 0.124 was shown in the question 'HPV vaccine can cause infertility'. The results from the study also showed a slight significance of 0.139 in the question 'there are health benefits for getting HPV vaccine'.

\section{Behaviour towards HPV Vaccine}

Results from the study show that $53 \%$ of respondents had immunised their daughters, 39\% had not while $8 \%$ were not sure whether the child have been immunised or not. All parents who did not immunise their daughters had intentions to do so. Data from the study shows that $21 \%$ of respondents' daughters had completed all the vaccine doses, $25 \%$ had not while $10 \%$ of the respondents were not sure whether the child has completed the doses or not. Almost all the respondents (98\%) had no cultural

\section{Virology \& Immunology Journal}

or spiritual beliefs that prevented them from having their daughters vaccinated. Respondents' behaviour towards HPV vaccine is illustrated in figure 5.

To test the relationship between the respondents' level of education and behaviour towards HPV vaccine, one-way ANOVA was used. $p$-value was calculated to evaluate the relationship. The results from the study revealed a significance of 0.381 for the question whether the child had been immunised. A significance of 0.491 was obtained for the question whether parents would encourage other parents to take their daughters for immunisation. The question whether there were cultural or religious beliefs that hindered HPV vaccination of their daughters obtained a significance of 0.140 . Table 2 shows the ANOVA results of respondents' level of education and behaviour.

Regarding the HPV vaccine uptake, the results show that $53 \%$ of the respondents' daughters we already vaccinated; $39 \%$ had not, and $8 \%$ were not sure whether their daughters had been vaccinated or not. Table 3 shows the age and immunisation status of the girls.

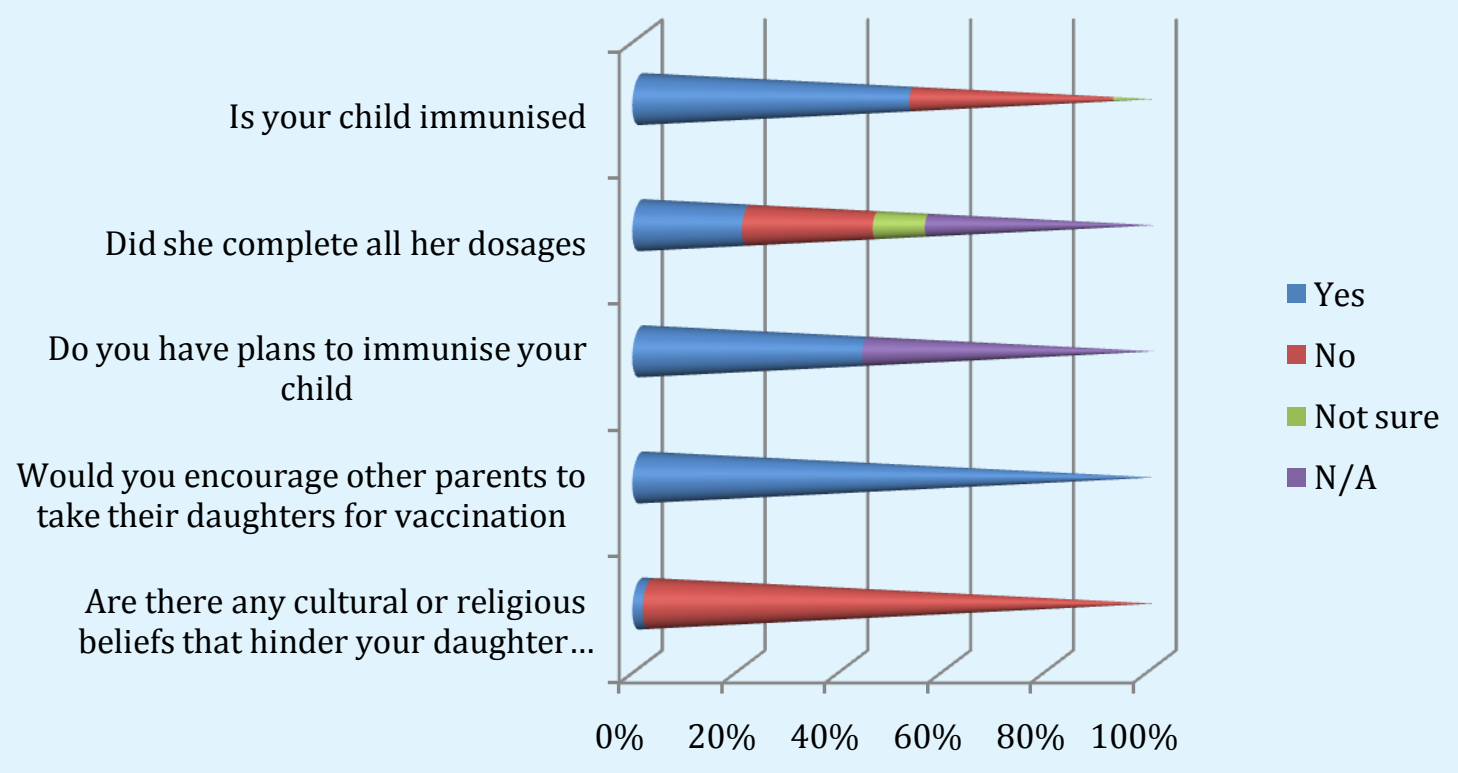

Figure 5: Respondents' behaviour towards the HPV vaccine. 


\begin{tabular}{|c|c|c|c|c|}
\hline Parameter & $\begin{array}{c}\text { Mean } \\
\text { square }\end{array}$ & Df & F & Sig \\
\hline Is your child immunised? & 0.425 & 3 & 1.035 & 0.381 \\
\hline $\begin{array}{c}\text { Would you encourage other } \\
\text { parents to take their } \\
\text { daughters for vaccination? }\end{array}$ & 0.008 & 3 & 0.811 & 0.491 \\
\hline $\begin{array}{c}\text { Are there any cultural or } \\
\text { religious beliefs that hinder } \\
\text { your daughter from taking } \\
\text { the HPV vaccine? }\end{array}$ & 0.036 & 3 & 1.869 & 0.14 \\
\hline
\end{tabular}

Table 2: Respondents' level of education and behaviour towards HPV vaccine.

\begin{tabular}{|c|c|c|c|c|}
\hline Age of child & Yes & No & Not sure & Total \\
\hline 9 years & 2 & 13 & 2 & 17 \\
\hline 10 years & 3 & 14 & 1 & 18 \\
\hline 11 years & 8 & 10 & 5 & 23 \\
\hline 12 years & 23 & 1 & 0 & 24 \\
\hline 13 years & 17 & 1 & 0 & 18 \\
\hline Total & 53 & 39 & 8 & 100 \\
\hline
\end{tabular}

Table 3: Age of child and immunisation status (HPV vaccine uptake).

Data from the study results indicated that of the respondents $65.1 \%$ knew about HPV infection; $86 \%$ knew about cervical cancer, and $64.5 \%$ knew about the HPV vaccine. Eighty-one $(81.5 \%)$ of the respondents had a positive attitude towards the HPV vaccine and $62.4 \%$ displayed a positive behaviour towards the HPV vaccine. Table 4.5 shows the overall results of the respondents knowledge, attitudes and behaviour towards HPV/HPV vaccine.

\begin{tabular}{|c|c|}
\hline Variable & Percentage (\%) \\
\hline Knowledge about HPV infection & 65.1 \\
\hline Knowledge about cervical cancer & 86 \\
\hline Knowledge about HPV vaccine & 64.5 \\
\hline Attitude towards HPV vaccine & 81.5 \\
\hline Behaviour towards HPV vaccine & 62.4 \\
\hline
\end{tabular}

Table 4: Respondents' overall knowledge, attitude and behaviour towards HPV/HPV vaccine.

\section{Discussion of Findings}

The purpose of the study was to determine the knowledge, attitude and behaviour of parents towards

\section{Virology \& Immunology Journal}

HPV and HPV vaccine in preventing cervical cancer in girls aged 9 to 13 years and HPV vaccine uptake.

\section{Knowledge about HPV Infection}

The results indicated that most (76\%) of the respondents had heard about HPV; 65.1\% had knowledge about HPV infection, and 68\% knew that HPV causes cervical cancer. Thus only a small percentage of respondents were not aware of HPV infection. Lack of knowledge of HVP infection could lead to the spread of HPV and leave young girls at risk of contracting HPV infection.

Seventy-one $(71 \%)$ of the respondents $(71 \%)$ knew that HPV can be prevented and were aware that having multiple sex partners put one at risk of contracting HPV infection thus exposing one to cervical cancer. This is demonstrated by respondents' willingness to vaccinate and complete the dosages for their daughters.

The level of knowledge displayed it implies that the parents would comply with the HPV prevention strategy since they are aware of HPV infection. The study also revealed that respondents with a higher educational level were more knowledgeable about HPV. This shows a positive relationship between the level of education and knowledge of HPV.

\section{Knowledge about Cervical Cancer}

The results from the study revealed high awareness and knowledge of cervical cancer. Almost (98\%) of the responds knew that cervical cancer is a serious disease and can pose a threat to the health of their daughters and 99\% knew that cervical cancer can cause death. Ninetyfour (94\%) of the respondents knew that cervical cancer is preventable and $93 \%$ knew that it can be treated if diagnosed early; most of the respondents (76\%) knew that a Pap smear is used for cervical cancer detection and 53\% knew the interval period for doing Pap smears. High awareness and knowledge about cervical cancer contributed to respondents' positive attitude towards HPV vaccine. The overall results indicate that $86 \%$ of the respondents are knowledgeable about cervical cancer. This implies that parents are most likely to comply with the HPV vaccine prevention strategy in order to prevent the disease.

\section{Knowledge about the HPV Vaccine}

The results in this study show a high awareness and knowledge of the HPV vaccine. This is shown by $86 \%$ the 


\section{Virology \& Immunology Journal}

respondents, who had heard of the HPV vaccine and $80 \%$ knew that it prevents cervical cancer. The results indicated an average of $64.5 \%$ knowledge about the HPV vaccine. Lower levels of knowledge were seen in dosage interval of the vaccine (30\%) and adverse effects (37\%.) Little knowledge of dosage intervals could result in girls missing or not completing doses. This could lead to reducing the government initiative of the prevention strategy against cervical cancer. Despite the little knowledge of vaccine doses and side effects parents believed that it is important to vaccinate their daughters.

High awareness of HPV vaccine was observed in study with $76.2 \%$ and $75.9 \%$ respectively $[4,9]$. In contrast, in study in Japan observed slightly lower levels of knowledge regarding HPV vaccine, where $52.5 \%$ of mothers had heard about HPV vaccine [10].

Another study also observed slightly lower awareness (52.7\%) of HPV vaccine [10]. The lower levels of awareness and knowledge of HPV vaccine in study could be because the respondents did not receive sufficient information (education) about HPV vaccine. However, despite low levels of awareness, most respondents showed a positive attitude to HPV vaccine as evidenced by their willingness to vaccinate their daughters [10-13].

\section{Attitude towards the HPV Vaccine}

In this study respondents showed positive attitude towards the HPV vaccine. All respondents agreed that there are health benefits for getting HPV vaccine, as shown by $78 \%$ strongly agreeing and $22 \%$ agreeing.

Respondents also agreed that HPV vaccine is effective in preventing cervical cancer revealed by $69 \%$ strongly agreed while $31 \%$ agreed. Of the respondents, $45 \%$ strongly agreed, $54 \%$ agreed and $1 \%$ disagreed that a girl should take all 3 doses.

Of the respondents, $52 \%$ strongly agreed, $46 \%$ agreed and only $2 \%$ disagreed that it is important to complete all 3 doses.

Thirty-eight percent (38\%) of the respondents disagreed and $2 \%$ strongly disagreed that HPV vaccine information was clearly explained.

These findings demonstrate positive attitudes of parents towards HPV vaccine and confirm that HPV vaccine was highly accepted if it became widely available [3]. The parents' positive attitude towards HPV vaccine resulted in a positive behaviour as evidenced by parents' willingness to vaccinate their daughters. This showed a positive relationship between attitudes and behaviour.

The study results affirm the factors or attributes of the Health Belief Model of perceived benefits and perceived vulnerability. The respondents perceived that there are health benefits of getting HPV vaccine to prevent cervical cancer and that cervical cancer is a serious disease and that it was a threat to their daughters' good health. These perceptions influenced the respondents' behaviour to accept the HPV vaccine (or to take their daughters for vaccination) so as to protect their daughters from the threat of cervical cancer. This also influenced parents compliance as shown by girls completing the doses.

The results demonstrated the relationship between education and attitudes as shown by a significance of 0.074 to the question 'HPV vaccine is effective in preventing cervical cancer. Significance was observed in the questions 'the girl should get all three doses of HPV vaccine' and 'it is important to complete all three doses'. The ANOVA test revealed that educational level influenced how respondents' answer these questions.

\section{Behaviour towards HPV Vaccine}

The results showed positive towards HPV vaccine. Of the respondents, 53\% had immunised their daughters; $21 \%$ had completed their dosages, and $99 \%$ would encourage other parents to take their daughters for vaccination.

Respondents with less HPV infection knowledge also had a positive behaviour towards the HPV vaccine and were willing to vaccinate their daughters. Some respondents had vaccinated their daughters, and finished the doses. Parents who did not vaccinate their daughters and those who did not receive all the doses expressed willingness to have their daughters to complete or take the vaccine. There was nothing hindering the parents from vaccinating their daughters as reported by $98 \%$ of the patents. The ANOVA showed no relationship between educational level and behaviour. All the respondents answered the same and showed the same behaviour regardless of the education level.

\section{Limitations}

This was self-sponsored study, there were some financial constraints. Most males were not willing to participate in the study, they believed it was for women 
since cervical cancer affects females which resulted in lower numbers of male respondents.

\section{Conclusion}

The results revealed that parents had some awareness and knowledge about HPV infection, cervical cancer, and the HPV vaccine. Parents showed positive attitudes towards HPV vaccine as shown by respondents' willingness to vaccinate their daughters. A positive behaviour was displayed by parents as evidenced by the number of girls vaccinated and some who completed their doses. Knowledge and a positive attitude are needed to maximise the uptake of HPV vaccine.

\section{Acknowledgements}

I would like to thank the respondents who participated in the study and the Ministry of Health for granting me the permission to conduct the study. I also extend my gratitude to University of South Africa for the continuous support and guidance.

\section{References}

1. Yu Y, Xu M, Sun J, Li R, Li M, et al. (2016) Human papillomavirus infection and vaccination: awareness and knowledge of HPV and acceptability of HPV vaccine among teenage daughters in Weihai, Shandong, China. PLoS ONE 11(1): e0146741.

2. Polit DF, Beck CT (2008) Nursing research: generating and assessing evidence for nursing practice. 8thedition. Philadelphia: Wolters Kluwer.

3. Turiho AK, Okello ES, Muhwezi WW, Harvey S, Byakika-Kibwika P, et al. (2014) Effect of schoolbased Human papillomavirus (HPV) vaccination on adolescent girls' knowledge and acceptability of the HPV vaccine in Ibanda in Uganda. African Journal of Reproductive Health 18(4): 45-53.

4. Haesebaert J, Lutringer-Magnin D, Kalecinski J, Barone G, Jacquard AC, et al. (2012) French women's knowledge of and attitudes towards cervical cancer prevention and the acceptability of HPV vaccination among those with 14-18 year old daughters: a quantitative-qualitative study. BMC Public Health 12: 1034.

\section{Virology \& Immunology Journal}

5. ICO (2014) HPV Information Centre on HPV and Cancer (Botswana). Human Papilloma and related cancers: fact sheet.

6. Hussain S, Nasare V, Kumari M, Sharma S, Khan MA, et al. (2014). Perception of Human Papilloma virus infection, cervical cancer and HPV vaccination in North Indian population. PLoS ONE 9(11): e112861.

7. Jaisamrarn U, Castellsague X, Garland SM, Naud P, Palmroth J, et al. (2013) Natural history of progression of HPV infection to cervical lesion or clearance: analysis of the control arm of the large randomised PATRICIA study. PLoS ONE 8(11): e79260.

8. Ramogola-Masire D (2014) HPV vaccine for cervical cancer prevention in Botswana. Commonwealth Health Partnerships.

9. Ayissi CA, Wamai RG, Oduwo GO, Perlman S, Welty E, et al. (2012) Awareness, acceptability and uptake of human papilloma virus vaccine among Cameroonian school-attending female adolescents. Journal of Community Health 37(6): 1127-1135.

10. Hanley SJ, Yoshioka E, Ito Y, Konno R, Hayashi Y, et al. (2012) Acceptance of and attitudes towards human papilloma virus vaccination in Japanese mothers of adolescent girls. Vaccine 30(39): 5740-5747.

11. Kornfeld J, Byrne MM, Vanderpool R, Shin S, Kobertz E (2013) HPV knowledge and vaccine acceptability among Hispanic fathers. Journal of Primary Prevention 34: 59-69.

12. Milimo MM, Daka E, Sikuyuba L, Nyirenda J, Ngoma C (2015) Knowledge and attitudes of parents/guardians towards uptake of Human Papilloma Virus (HPV) vaccine in preventing cervical cancer among girls in Zambia. Merit Research Journals 3(3): 84-91.

13. Mouallif M, Bowyer HL, Festali S, Albert A, FilaliZegzouti Y, et al. (2014) Cervical cancer and HPV: awareness and vaccine acceptability among parents in Morocco. Vaccinate 32(3): 409-416. 